\title{
A Brief Note About Rott Contraction
}

\author{
EDUARDO L. FERMÉ and RICARDO O. RODRIGUEZ, \\ Departamento de Computación, Universidad de Buenos Aires, Pabellón \\ I Ciudad Universitaria (1428), Buenos Aires, Argentina. \\ E-mail: \{ferme,ricardo\}@dc.uba.ar
}

\begin{abstract}
One of the ways to model contraction functions for belief sets is epistemic entrenchment. The first step was provided by Gärdenfors in [5] function in terms of it and related the latter with the AGM contraction function. Later Hans Rott in [16] presented an entrenchment based contraction function that deeps not satisfy recovery. In this paper we provide an axiomatic characterization of Rott Contraction.' $\mathbf{L}_{\mathbf{L}}$
\end{abstract}

Keywords: AGM, belief contraction, recovery postulate, Rott contraction.

\section{Introduction}

"Even if all sentences in a knowledge set are accepted or considered as facts, this does not means that all sentences are of equal value for planning or problem solving purposes. Certain pieces of knowledge and belief about the world are more important than others when planning future actions, conducting scientific investigations or reasoning in general. We will say that some sentences in a knowledge system have a higher degree of epistemic entrenchment than others. The degree of entrenchment will, intuitively, have a bearing on what is abandoned from a knowledge set and what is retained, when a contraction or revision is carried out." [6, $\left.{ }_{1}\right]$

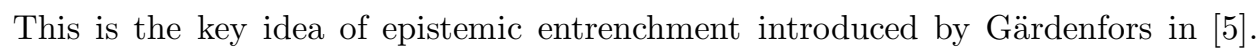
Using epistemic entrenchment, Gärdenfors defines an operation of contraction that

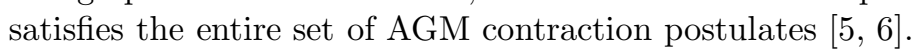

However, epistemic entrenchment also allows us to define entrenchment based contraction functions that do not satisfy all the AGM postulates. One of the more important such functions in the literature was proposed by Hans Rott in [1] ${ }^{1}$ ] and has been called Rott contraction. This contraction differs from the Gärdenfors contraction in not satisfying the controversial postulate of recovery. For discussions of

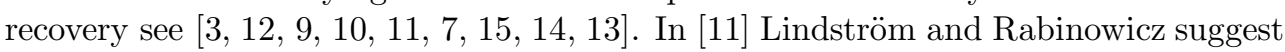
that any realistic entrenchment-based contraction operator should lie between those of Rott and Gärdenfors.

${ }^{1}$ Full version of a contributed paper presented at the 4 th Workshop on Logic, Language, Information and Computation (WoLLIC'97), http://www.di.ufpe.br/ wollic97, held in Fortaleza (Ceará), Brazil, August 19-22 1997, with scientific sponsorship by IGPL, FoLLI and ASL, and organised by Univ. Federal do Ceará (UFC) and Univ. Federal de Pernambuco (UFPE).

L. J. of the IGPL, Vol. 6 No. 6, pp. 
We give here an axiomatic characterization for Rott's entrenchment-based contraction operator, i.e. a characterization of the lower limit of what Lindström and Rabinowicz regard as reasonable contraction.

In Section $\overline{2}_{1}^{-1}$ we recall $A G M$ contraction functions, introduce epistemic entrenchment, relate it to AGM contraction, and also present Rott contraction. In Section we provide the promised axiomatic characterization of Rott contraction. Proofs are in the appendix.

After this work was finished we were informed that another axiomatic characterization has been independently obtained by Hans Rott and Maurice Pagnucco and will be published in a joint paper by these authors [i] $\bar{z}^{\prime}$.

\section{Background}

\subsection{AGM contraction}

The logic of theory change was introduced into philosophical logic and artificial intelligence a little over a decade ago. The initial step was provided by Alchourrón, Gärdenfors and Makinson in (1) (commonly called the AGM model). Basically, in the AGM model the beliefs of a rational agent are represented by a belief set $\mathbf{K}$, closed under logical consequence $C n$, where $C n$ satisfies the Tarski conditions $\mathbf{K} \subseteq C n(\mathbf{K})$ for any set $\mathbf{K}$ of propositions, $C n(C n(\mathbf{K})) \subseteq C n(\mathbf{K})$ and $C n(\mathbf{K}) \subseteq C n(\mathbf{H})$ if $\mathbf{K} \subseteq \mathbf{H}$ . We assume that $C n$ includes classical logical consequence, satisfies the rule of introduction of disjunction into premises and is compact. We write $\vdash x$ for $x \in \operatorname{Cn}(\emptyset)$.

A theory is understood to be any set $\mathbf{K}$ of proposition closed under $C n$, i.e. such that $C n(\mathbf{K})=\mathbf{K}$.

Let $\mathbf{L}$ be the set of all the sentences of the language. Let $\mathcal{K}$ the set of all theories of the language. The expansion function + from $\mathcal{K} \times \mathbf{L}$ to $\mathcal{K}$ is defined by $(\mathbf{K}+x)=$ $C n(\mathbf{K} \cup\{x\})$. The postulates for the AGM contraction are:

$$
\begin{array}{lll}
(\mathbf{K}-\mathbf{1}) & \mathbf{K}-x \text { is a theory whenever } \mathbf{K} \text { is a theory } & \text { (closure) } \\
(\mathbf{K}-\mathbf{2}) & \mathbf{K}-x \subseteq \mathbf{K} & \text { (inclusion) } \\
(\mathbf{K}-\mathbf{3}) & \text { If } x \notin \mathbf{K} \text {, then } \mathbf{K}-x=\mathbf{K} & \text { (vacuity) } \\
(\mathbf{K}-\mathbf{4}) & \text { If } x \notin C n(\emptyset), \text { then } x \notin \mathbf{K}-x & \text { (success) } \\
(\mathbf{K}-\mathbf{5}) & \text { If } x \leftrightarrow y \in C n(\emptyset) \text { then } \mathbf{K}-x=\mathbf{K}-y & \text { (extensionality) } \\
(\mathbf{K}-\mathbf{6}) & \mathbf{K} \subseteq(\mathbf{K}-x)+x \text { whenever } \mathbf{K} \text { is a theory } & \text { (recovery) } \\
(\mathbf{K}-\mathbf{7}) & \mathbf{K}-x \cap \mathbf{K}-y \subseteq \mathbf{K}-(x \wedge y) & \text { (conjunctive overlap) } \\
(\mathbf{K}-\mathbf{8}) & \text { If } x \notin \mathbf{K}-(x \wedge y), \text { then } \mathbf{K}-(x \wedge y) \subseteq \mathbf{K}-x & \text { (conjunctive inclusion). }
\end{array}
$$

\subsection{Epistemic entrenchment}

The idea of entrenchment for theories was introduced by Gärdenfors in [n to represent formally a preference ordering between formulae in a theory. He attempted to define the contraction of a theory by a sentence in terms of an order of the sentences, and identify the properties that the order must satisfy for the generated 
contraction to satisfy the AGM postulates.

Gärdenfors proposed the following set of axioms for the order among sentences:

(EE1) If $x \leq_{\mathbf{K}} y$ and $y \leq_{\mathbf{K}} z$, then $x \leq_{\mathbf{K}} z$

(transitivity)

(EE2) If $x \vdash y$, then $x \leq_{\mathbf{K}} y$

(dominance)

(EE3) $\quad x \leq_{\mathbf{K}}(x \wedge y)$ or $y \leq_{\mathbf{K}}(x \wedge y)$

(conjunctiveness)

(EE4) If $\mathbf{K} \neq \mathbf{K}_{\perp}$, then $x \notin \mathbf{K}$ if and only if $x \leq_{\mathbf{K}} y$ for all $y$

(minimality)

(EE5) If $y \leq_{\mathbf{K}} x$ for all $y$, then $\vdash x$

(maximality)

A relation satisfying (EE1) - (EE5) is a standard entrenchment ordering. Furthermore, he showed that entrenchment orderings can be connected with contraction functions by the following equivalences ${ }_{2}^{2}$ :

$(C \leq) \quad x \leq_{\mathbf{K}} y$ if and only if $x \notin \mathbf{K}-(x \wedge y)$ or $\vdash(x \wedge y)$.

Gärdenfors' entrenchment-based contraction

$(-G) \quad y \in \mathbf{K}-x$ if and only if $y \in \mathbf{K}$ and, either $\vdash x$ or $x<_{\mathbf{K}}(x \vee y)$.

Observation 2.1 ( $([\overline{5}, \overline{6}, \bar{j}])$ Let $\leq_{\mathbf{K}}$ be a standard entrenchment ordering on a consistent belief set $\mathbf{K}$. Furthermore let $-_{G}$ be the Gärdenfors entrenchment-based contraction on $\mathbf{K}$ defined by condition $\left(-{ }_{G}\right)$ from $\leq_{\mathbf{K}}$. Then $-_{G}$ satisfies the eight AGM postulates, and $(C \leq)$ also holds.

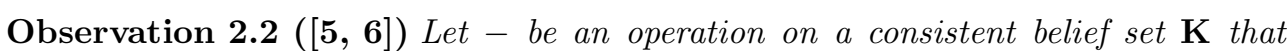
satisfies the eight $A G M$ postulates. Furthermore let $\leq_{\mathbf{K}}$ be the relation defined from - by condition $(C \leq)$. Then $\leq_{\mathbf{K}}$ satisfies the standard entrenchment postulates and $(-G)$ also holds.

Hans Rott $\left[1 \overline{1}{ }_{i}\right]$ has remarked that the comparison $x<_{\mathbf{K}}(x \vee y)$ is not intuitive, and proposed the following alternative definition of a contraction operation from an entrenchment ordering:

Rott's entrenchment-based contraction

$\left(-{ }_{R}\right) \quad y \in \mathbf{K}-x$ if and only if $y \in \mathbf{K}$ and, either $\vdash x$ or $x<_{\mathbf{K}} y$.

Rott also provided the following result:

Observation 2.3 Let $\leq_{\mathbf{K}}$ be a standard entrenchment ordering on a consistent belief set $\mathbf{K}$. Furthermore let $-_{R}$ be the Rott entrenchment-based contraction on $\mathbf{K}$ defined by condition $\left(-_{R}\right)$ from $\leq_{\mathbf{K}}$. Then $-_{R}$ satisfies all the AGM postulates except recovery.

Rott $\left[16\right.$, p. 169] proved that for all $x, \mathbf{K}-{ }_{\mathrm{R}} x \subseteq \mathbf{K}-{ }_{\mathrm{G}} x$. Lindström and Rabinowicz [1] 1. have proposed that a reasonable entrenchment based contraction operation should lie between Rott's operation and Gärdenfors' operation, in the sense that $\mathbf{K}-{ }_{\mathrm{R}} x \subseteq \mathbf{K}-x \subseteq \mathbf{K}-{ }_{\mathrm{G}} x$.

\footnotetext{
${ }^{2}$ We write $x<_{\mathbf{K}} y$ to denote $x \leq_{\mathbf{K}} y$ and $y \not_{\mathbf{K}} x$.
} 


\section{An axiomatic characterization of Rott contraction}

In section 12.2 we recalled that Hans Rott proved that his contraction satisfies all the AGM postulates except recovery. In this section we provide it with an axiomatic characterization. We make use of the following postulates:

$$
\text { If } \mathbf{K}-(x \wedge y) \subseteq \mathbf{K}-y \text { then } y \notin \mathbf{K}-x \text { or } \vdash x \text { or } \vdash y \quad \begin{aligned}
& \text { (Converse Conjunctive } \\
& \text { Inclusion) }
\end{aligned}
$$

If $x \in C n(\emptyset)$ then $\mathbf{K}-x=\mathbf{K} \quad$ (Failure) [4]

If $x \notin \mathbf{K}-y$ then $\mathbf{K}-y \subseteq \mathbf{K}-x \quad$ (Strong Inclusion)

Now we can characterize Rott Contraction in terms of postulates:

\section{Theorem 3.1}

1. Let $\leq_{\mathbf{K}}$ be a standard entrenchment ordering on a consistent belief set $\mathbf{K}$. Furthermore, let $-_{R}$ be Rott's entrenchment-based contraction on $\mathbf{K}$, defined from $\leq_{\mathbf{K}}$ by condition $\left(-_{\mathrm{R}}\right)$. Then $-_{R}$ satisfies closure, inclusion, success, extensionality, conjunctive overlap, failure, strong inclusion and converse conjunctive inclusion, and $(C \leq)$ also holds.

2. Let - be an operation on a consistent belief set $\mathbf{K}$ that satisfies closure, inclusion, success, extensionality, conjunctive overlap, failure, strong inclusion and converse conjunctive inclusion. Furthermore let $\leq_{\mathbf{K}}$ be the relation that is defined fromby $(C \leq)$. Then $\leq_{\mathbf{K}}$ satisfies the standard entrenchment postulates, and $\left(-_{R}\right)$ also holds.

Other interesting postulates are:

$$
\begin{aligned}
& \text { If } \forall x \text { and } \forall y \text {, then either } x \notin \mathbf{K}-y \text { or } y \notin \mathbf{K}-x \quad \text { (Expulsiveness) } \\
& \mathbf{K}-(x \wedge y)=\mathbf{K}-x \text { or } \mathbf{K}-(x \wedge y)=\mathbf{K}-y \quad \text { (Linear Hierarchical Ordering) } \\
& \mathbf{K}-y \subseteq \mathbf{K}-x \text { or } \mathbf{K}-x \subseteq \mathbf{K}-y \quad \text { (Linearity) }
\end{aligned}
$$

The following relations hold:

Observation 3.2 Let $\mathbf{K}$ be a belief set and - an operator from $\mathcal{K} \times \mathbf{L}$ to $\mathcal{K}$. Then:

1. If - satisfies strong inclusion then it satisfies conjunctive inclusion.

2. If - satisfies inclusion, failure and strong inclusion then it satisfies vacuity.

3. If - satisfies closure, success and strong inclusion then it satisfies expulsiveness. 
4. If - satisfies inclusion, failure, strong inclusion and expulsiveness then it satisfies linearity.

5. If - satisfies closure, success, extensionality and strong inclusion then it satisfies linear hierarchical ordering.

\section{Acknowledgements}

We would like to thank Sven Ove Hansson and David Makinson for valuable comments on an the earlier version of this paper. We are also indebted to the anonymous referees for corrections, suggestions and incisive remarks on a draft.

\section{Appendix: Proofs}

\section{Proof of Theorem 'ỉ.}

Part 1: Closure, inclusion, success, extensionality and conjunctive overlap follow from observation 2.3.

Converse conjunctive inclusion: Let $\mathbf{K}-(x \wedge y) \subseteq \mathbf{K}-y$. We have two cases: 1 . $y \in \mathbf{K}-y$ : It follows from success that $\vdash y$. 2. $y \notin \mathbf{K}-y$ : then $y \notin \mathbf{K}-(x \wedge y)$. It follows from $\left(-{ }_{R}\right)$ that $y \notin \mathbf{K}$ or $\left(y \leq_{\mathbf{K}} x \wedge y\right.$ and $\left.\forall x \wedge y\right)$. We have two subcases 2.1. $y \notin \mathbf{K}$ : then by inclusion (see observation 2.3i) $y \notin \mathbf{K}-x$. 2.2. $y \in \mathbf{K}$ : then $y \leq_{\mathbf{K}} x \wedge y$. By $(\mathbf{E E 2}) x \wedge y \leq_{\mathbf{K}} x$ and by $(\mathbf{E E} 1) y \leq_{\mathbf{K}} x$ hence by $\left(-{ }_{R}\right) y \notin \mathbf{K}-x$ or $\vdash x$.

Failure: It follows trivially, since if $\vdash x$, then by $\left(-{ }_{R}\right) y \in \mathbf{K}-x$ if and only if $y \in \mathbf{K}$.

Strong inclusion: Let $x \notin \mathbf{K}-y$. By $(-R) x \notin \mathbf{K}$ or $\left(\forall y\right.$ and $\left.x \leq_{\mathbf{K}} y\right)$. We have two subcases: 1. $x \notin \mathbf{K}$ : then $\mathbf{K}-x=\mathbf{K}$ (since by observation 2.3, satisfies vacuity); then $\mathbf{K}-y \subseteq \mathbf{K}-x$ (since by observation 2.3 , - satisfies inclusion). 2. $x \in \mathbf{K}$ : then $\left(\forall y\right.$ and $\left.x \leq_{\mathbf{K}} y\right)$ Let $z \in \mathbf{K}-y$, then (by $\left.\left(-{ }_{R}\right)\right) z \in \mathbf{K}$ and $y<_{\mathbf{K}} z$. By (EE1) $x<_{\mathbf{K}} z$ then by $(-R) z \in \mathbf{K}-x$ hence $\mathbf{K}-y \subseteq \mathbf{K}-x$.

$(C \leq)^{3:}$ : For one direction let $x \leq_{\mathbf{K}} y$ and $x \in \mathbf{K}-(x \wedge y)$. We need to prove $\vdash x \wedge y$. By $(-R)$ we have: $x \in \mathbf{K}-(x \wedge y)$ if and only if $x \in \mathbf{K}$ and either $\vdash x \wedge y$ or $x \wedge y<_{\mathbf{K}} x$. Therefore: $\vdash x \wedge y$ or $x \wedge y<_{\mathbf{K}} x$. Let $x \wedge y<_{\mathbf{K}} x$ : then, by (EE3), $y \leq_{\mathbf{K}} x \wedge y$; and since $x \leq_{\mathbf{K}} y$, we have by $(\mathbf{E E 1})$ that $x \leq_{\mathbf{K}}(x \wedge \mathrm{y})$, contradiction, hence $\vdash x \wedge y$.

For the second direction we have two subcases: $1 . x \notin \mathbf{K}-(x \wedge y)$ : Then by $\left(-{ }_{R}\right)$, $x \notin \mathbf{K}$ or $\forall x$ and $x \leq_{\mathbf{K}} x \wedge y$. If $x \notin \mathbf{K}, x \leq_{\mathbf{K}} y$ follows (by (EE4)). If $x \leq_{\mathbf{K}} x \wedge y$, by $\left(\mathbf{E E 1}\right.$ ), (since by $\left.(\mathbf{E E 2}) x \wedge y \leq_{\mathbf{K}} y\right), x \leq_{\mathbf{K}} y \cdot 2 . \vdash x \wedge y$, then $\vdash y$, hence by $(\mathbf{E E 2}), x \leq_{\mathbf{K}} y$. This completes the proof.

${ }^{3}$ The idea for this proof was provided by an anonymous referee. 
Part 2: (EE2) - (EE5) are proved by Gärdenfors and Makinson from closure, inclusion, success, extensionality, failure and $(C \leq)$ in |'ini|, pp. 93-94.

- (EE1)

We demonstrate by reductio ad absurdum. Let $x \leq_{\mathbf{K}} y, y \leq_{\mathbf{K}} z$ and $x \mathbb{Z}_{\mathbf{K}} z$. It follows by $(C \leq)$ that: (a) either $\vdash x \wedge y$ or $x \notin \mathbf{K}-(x \wedge y)$; (b) either $\vdash y \wedge z$ or $y \notin \mathbf{K}-(y \wedge z)$; and (c) $\forall x \wedge z$ and $x \in \mathbf{K}-(x \wedge z)$.

1. Let $\vdash x \wedge y$ : then $\vdash x$ and $\vdash y$. By closure $y \in \mathbf{K}-(y \wedge z)$, then by condition (b) $\vdash y \wedge z$, so $\vdash z$, and $\vdash x \wedge z$; contradiction.

2. Let $\vdash y \wedge z$ : then $\vdash y$ and $\vdash z$. By closure $z \in \mathbf{K}-(x \wedge z)$, then by condition (c) and closure $x \wedge z \in \mathbf{K}-(x \wedge z)$; hence by success $\vdash x \wedge z$; contradiction.

By 1. and 2. (a), (b) and (c) are reduced to $x \notin \mathbf{K}-(x \wedge y), y \notin \mathbf{K}-(y \wedge z), \forall x \wedge z$ and $x \in \mathbf{K}-(x \wedge z)$.

3. By strong inclusion $\mathbf{K}-(x \wedge y) \subseteq \mathbf{K}-x$, then by converse conjunctive inclusion we have $\vdash x$ or $\vdash y$ or $x \notin \mathbf{K}-y$. But since $x \notin \mathbf{K}-(x \wedge y)$ and $y \notin \mathbf{K}-(y \wedge z)$, by closure $\forall x$ and $\forall y$. Then $x \notin \mathbf{K}-y$ and by strong inclusion we have $\mathbf{K}-y \subseteq \mathbf{K}-x$.

4. $y \notin \mathbf{K}-(y \wedge z)$ implies by strong inclusion that $\mathbf{K}-(y \wedge z) \subseteq \mathbf{K}-y$, then by converse conjunctive inclusion we have $\vdash y$ or $\vdash z$ or $y \notin \mathbf{K}-z$; by closure $\forall y$ and $\forall z$ (since by success, closure and condition $(\mathbf{C}), z \notin \mathbf{K}-(x \wedge z)$ ). Then $y \notin \mathbf{K}-z$ and by strong inclusion we have $\mathbf{K}-z \subseteq \mathbf{K}-y$.

5. It follows from success that $z \notin \mathbf{K}-z$, so by closure $(x \wedge z) \notin \mathbf{K}-z$; and since $z \notin \mathbf{K}-(x \wedge z)$ we obtain by strong inclusion that $\mathbf{K}-z=\mathbf{K}-(x \wedge z)$. So $x \in \mathbf{K}-(x \wedge z)=\mathbf{K}-z \subseteq \mathbf{K}-y \subseteq \mathbf{K}-x$. Hence by success $\vdash x$; contradiction.

$\bullet-R$

$\Rightarrow)$

Let $y \in \mathbf{K}-x$ and $\forall x$. It follows by inclusion that $y \in \mathbf{K}$. We have two cases: 1. $\vdash y$ : By closure $y \in \mathbf{K}-(x \wedge y)$, then by $(C \leq), y \mathbb{Z}_{\mathbf{K}} x$. By success and closure $x \notin \mathbf{K}-(x \wedge y)$; then, by $(C \leq), x \leq_{\mathbf{K}} y$. Hence $x<_{\mathbf{K}} y$.

2. $\forall y$ : By converse conjunctive inclusion $\mathbf{K}-(x \wedge y) \nsubseteq \mathbf{K}-y$, then by strong inclusion $y \in \mathbf{K}-(x \wedge y)$; then by $(C \leq), y \not \mathbf{K} x$. By success and closure $x \notin \mathbf{K}-(x \wedge y)$; then, by $(C \leq), x \leq_{\mathbf{K}} y$. Hence $x<_{\mathbf{K}} y$.

$\Leftarrow)$

1. Let $y \in \mathbf{K}$ and $\vdash x$. By failure $\mathbf{K}-x=\mathbf{K}$ then $y \in \mathbf{K}-x$.

2. Let $y \in \mathbf{K}$ and $x<_{\mathbf{K}} y$. By $(C \leq) x \notin \mathbf{K}-(x \wedge y)$, then by strong inclusion $\mathbf{K}-(x \wedge y)$. For reductio ad absurdum let $y \notin \mathbf{K}-x$; then $y \notin \mathbf{K}-(x \wedge y)$ then by $(C \leq), y \leq_{\mathbf{K}} x$. Contradiction. 
Proof of Observation

1. If - satisfies strong inclusion then it satisfies conjunctive inclusion: Let $x \notin \mathbf{K}-(x \wedge y)$. Then by strong inclusion $\mathbf{K}-(x \wedge y) \subseteq \mathbf{K}-x$.

2. If - satisfies inclusion, failure and strong inclusion then it satisfies vacuity: Let $x \notin \mathbf{K}$ and $\vdash y$. Then by failure $x \notin \mathbf{K}-y=\mathbf{K}$; by strong inclusion $\mathbf{K}=\mathbf{K}-y \subseteq \mathbf{K}-x$. Hence by inclusion $\mathbf{K}-x=\mathbf{K}$.

3. If - satisfies closure, success and strong inclusion then it satisfies expulsiveness: Let $\forall x$ and $\forall y$. By closure and success $x \wedge y \notin \mathbf{K}-x$ and $x \wedge y \notin \mathbf{K}-y$, then by strong inclusion $\mathbf{K}-x \subseteq \mathbf{K}-(x \wedge y)$ and $\mathbf{K}-y \subseteq \mathbf{K}-(x \wedge y)$. Let $y \in \mathbf{K}-x$, then $y \in \mathbf{K}-(x \wedge y)$, then by success $x \notin \mathbf{K}-(x \wedge y)$; hence $x \notin \mathbf{K}-y$.

4. If - satisfies inclusion, failure, strong inclusion and expulsiveness then it satisfies linearity:

If $\vdash x$ then by failure $\mathbf{K}-x=\mathbf{K}$, and by inclusion $\mathbf{K}-y \subseteq \mathbf{K}-x$. By the same reasoning if $\vdash y$ then $\mathbf{K}-x \subseteq \mathbf{K}-y$. Let $\forall x$ and $\forall y$, then by expulsiveness $y \notin \mathbf{K}-x$ or $x \notin \mathbf{K}-y$. Hence by strong inclusion $\mathbf{K}-x \subseteq \mathbf{K}-y$ or $\mathbf{K}-y \subseteq \mathbf{K}-x$.

5. If - satisfies closure, success, extensionality and strong inclusion then it satisfies linear hierarchical ordering:

If $\vdash x$ or $\vdash y$ then $\vdash(x \wedge y) \leftrightarrow x$ or $\vdash(x \wedge y \leftrightarrow y)$, then by extensionality $\mathbf{K}-(x \wedge y)=\mathbf{K}-x$ or $\mathbf{K}-(x \wedge y)=\mathbf{K}-y$. Let $\forall x$ and $\forall y$. By closure and success $x \wedge y \notin \mathbf{K}-x$ and $x \wedge y \notin \mathbf{K}-y$, then by strong inclusion $\mathbf{K}-x \subseteq \mathbf{K}-(x \wedge y)$ and $\mathbf{K}-y \subseteq \mathbf{K}-(x \wedge y)$. By success $x \notin \mathbf{K}-(x \wedge y)$ or $y \notin \mathbf{K}-(x \wedge y)$. Then by strong inclusion $\mathbf{K}-x \subseteq \mathbf{K}-(x \wedge y)$ or $\mathbf{K}-y \subseteq \mathbf{K}-(x \wedge y)$ Hence $\mathbf{K}-(x \wedge y)=\mathbf{K}-x$ or $\mathbf{K}-(x \wedge y)=\mathbf{K}-y$.

\section{References}

[1] Carlos Alchourrón, Peter Gärdenfors, and David Makinson. On the logic of theory change: Partial meet contraction and revision functions. Journal of Symbolic Logic, 50:510-530, 1985.

[2] Carlos Alchourrón and David Makinson. On the logic of theory change: Contraction functions and their associated revision functions. Theoria, 48:14-37, 1982.

[3] Eduardo Fermé. Five faces of recovery. (manuscript), 1998.

[4] André Fuhrmann and Sven Ove Hansson. A survey of multiple contraction. Journal of Logic, Language and Information, 3:39-76, 1994.

[5] Peter Gärdenfors. Knowledge in Flux: Modeling the Dynamics of Epistemic States. MIT Press, Cambridge, 1988.

[6] Peter Gärdenfors and David Makinson. Revisions of knowledge systems using epistemic entrenchment. In Moshe Y. Vardi, editor, Proceedings of the Second Conference on Theoretical Aspects of Reasoning About Knowledge, pages 83-95, Monterey, California, March 1988. Morgan Kaufmann.

[7] Sven Ove Hansson. Belief contraction without recovery. Studia Logica, 50:251-260, 1991.

[8] Sven Ove Hansson. A Textbook of Belief Dynamics. Kluwer Academic Publisher, Dordrecht, In Press.

[9] Isaac Levi. The fixation of belief and its undoing: changing beliefs through inquiry. Cambridge University Press, Cambridge, 1991. 
[10] IsaAC Levi. Contraction and informational value. (manuscript, fifth version), August 1997.

[11] Sten Lindström and Wlodzimierz Rabinowicz. Epistemic entrenchment with incomparabilities and relational belief revision. In Fuhrmann and Morreau, editors, The Logic of Theory Change, pages 93-126, Berlin, 1991. Springer-Verlag.

[12] David Makinson. On the status of the postulate of recovery in the logic of theory change. The Journal of Philosophical Logic, 16:383-394, 1987.

[13] David Makinson. On the force of some apparent counterexamples to recovery. In E. Garzón Valdéz et all, editor, Normative Systems in Legal and Moral Theory: Festschrift for Carlos Alchourrón and Eugenio Bulygin, pages 475-481, Berlin, 1997. Duncker \& Humblot.

[14] Abhaya Nayak. Foundational belief change. Journal of Philosophical Logic, 23:495-533, 1994.

[15] Reinhard Niederée. Multiple contraction. a further case against Gärdenfors' principle of recovery. In Fuhrmann and Morreau, editors, The Logic of Theory Change, pages 322-334, Berlin, 1991. Springer-Verlag.

[16] Hans Rott. Two methods of constructing contractions and revisions of knowledge systems. Journal of Philosophical Logic, 20:149-173, 1991.

[17] Hans Rott and Maurice Pagnucco. Severe withdrawal (and recovery). (manuscript), 1997.

Received 4 October 1997. Revised 6 May 1998 
Copyright of Logic Journal of the IGPL is the property of Oxford University Press / USA and its content may not be copied or emailed to multiple sites or posted to a listserv without the copyright holder's express written permission. However, users may print, download, or email articles for individual use. 
Copyright (C1998 Professor Dov Gabbay. The Logic Journal of the IGPL is published under license from Professor Dov Gabbay as owner. Professor Dov Gabbay is the founding editorin- chief of The Logic Journal of the IGPL. Logic Journal of the IGPL content may not be copied or emailed to multiple sites or posted to a listserv without the copyright holder's express written permission. However, users may print, download, or email articles for individual use. 